\title{
What anesthesiologists need to know about mesenteric traction syndrome
}

\begin{abstract}
The first time I heard of mesenteric traction syndrome was three years into my training as an anesthesia resident. This first time was unfortunately intraoperatively while my patient was acutely unstable and mesenteric traction syndrome was part of my anesthesia team's differential diagnosis. Following this event I set out to educate myself about mesenteric traction syndrome and was shocked to find there was very little mention of it in the anesthesia literature, especially common textbooks that I had used throughout my residency to educate myself. The goal of this article is to present the literature on mesenteric traction that I encountered during my research and to provide a thorough review of the consensus on the etiology, pathophysiology, prevention and treatment of mesenteric traction syndrome. At the end of the review, I examine areas of mesenteric traction that need further investigation in order to fully understand mesenteric traction syndrome and what we can do, as anesthesiologists, to prevent it and appropriately treat it.
\end{abstract}

Volume 8 Issue 3 - 2017

Brenda J Satterthwaite, Ibtesam Hilmi

University of Pittsburgh Medical Center, USA

\begin{abstract}
Correspondence: Ibtesam A Hilmi, Professor of Anesthesiology, Clinical and Translational Science Institute, University of Pittsburgh Medical Center, Department of Anesthesiology, C-Wing, Suite 200, 200 Lothrop Street Pittsburgh, PA 15213, USA, Tel 412-647-6232, Email hilmiia@anes.upmc.edu
\end{abstract}

Received: June 26, 2017 | Published: June 29, 2017

\section{Introduction}

After 3 years of training in anesthesiology I had never heard the words mesenteric traction syndrome (MTS). Unfortunately, the first time I was introduced to this term occurred while taking care of a patient when he suddenly became severely hemodynamically unstable. The case was a pancreaticoduodenectomy being performed on a 63 year old male with locally advanced pancreatic cancer. The patient had undergone two rounds of abraxane and gemcitabine chemotherapy prior to the surgery. His comorbidities included coronary artery disease with previous five vessel CABG and ascending aortic aneurysm repair 6 years ago, hypertension, hyperlipidemia, and bipolar disorder. He was a current pack per day smoker but denied any alcohol or drug use. His pertinent home medications included Xanax, aspirin which had been stopped 7 days prior, lithium, metoprolol, oxycontin, and oxycodone. He denied any previous anesthesia-related problems. A regadenoson stress test was performed 3 months prior to surgery; results were negative for ischemia and showed an ejection fraction of $74 \%$. An EKG performed a week prior showed normal sinus rhythm with a first degree AV nodal block.

Preoperatively the patient was prescribed oral gabapentin 600 $\mathrm{mg}$ and oxycontin $30 \mathrm{mg}$ as part of an enhanced recovery after surgery (ERAS) protocol. The patient was induced with ketamine, propofol, succhinylcholine, endotracheal tube was placed without difficulty. A post induction arterial line was placed in the right radial artery and triple-lumen central line was inserted in his right internal jugular. As part of the ERAS protocol the patient was started on lidocaine, ketamine, and magnesium infusions along with 0.5 MAC of sevoflurane. Surgery was started and the initial arterial blood gas test showed normal values. Two hours into the surgery the patient's blood pressure dropped dramatically, from a systolic pressure of 120 $\mathrm{mmHg}$ to $50 \mathrm{mmHg}$ within a 20 second period. A total of $400 \mathrm{mcg}$ of phenylephrine was given to the patient without any significant response.

Over the next 20 minutes the patient received a total of $400 \mathrm{mcg}$ phenylephrine, 17 units of vasopressin, and $150 \mathrm{mcg}$ of epinephrine. He was started on norepinephrine and vasopressin infusions and received one liter of crystalloid and one liter of colloid solution.
His blood pressure eventually began to stabilize after 15 minutes of treatment with vasopressors and administration of intravenous fluid.

As blood pressure management was underway, a quick differential diagnosis flowed through my head:

a. Was this surgical? There was neither bleeding in the surgical field, nor evidence of inferior vena cava compression.

b. Could this be pulmonary in origin? Could this be an air embolus (from an opened vessel) or clot embolus? However, the end-tidal $\mathrm{CO} 2$, oxygen saturation and peak airway pressures remained unchanged from baseline and were all within normal limits

c. Could this be cardiac in origin given patient's history of previous cardiac problems? To rule out such possibility, a transesophageal echocardiogram probe was placed which showed normal wall motion of the right and left ventricles, adequate ventricular filling, and normal valvular function, no right ventricular strain, and no tricuspid valve regurgitation which eliminated the possibility of significant pulmonary embolism.

d. Could this an allergic reaction to one of the medications that we used? Knowing that the most common offenders in the etiology of intraoperative allergic reaction are muscle relaxants and antibiotics. However, the patient had received antibiotics over two hours prior to this incident and the last dose of rocuronium was 40 minutes prior, making both medications unlikely suspects. There were also no signs or symptoms of allergic reaction (urticarial rash, bronchospasm), which all together made the possibility of drug-induced reaction very unlikely.

With the most common causes of acute hypotension being ruled out, my attending and I started to think of other possible etiologies. My attending suggested the possibility of MTS, which was the initial time I had heard this term. Neither of us had a clear understanding of how to treat MTS besides of supportive therapy. Luckily our patient's hemodynamic instability improved after 25 minutes of intense resuscitation. He was extubated three hours after the conclusion of the surgery and discharged home four days later without any complications. 
Following my experience with this case I decided to perform a literature search on MTS, I found multiple articles published over the last 30 years on MTS. Interestingly, most of the publications were in the surgical journals and with very little mentioning about MTS in anesthesiology literatures. I found this very odd since it is the anesthesiologists who have to deal with intraoperative cardiovascular instability. The goal of this article is to review the publications that I found most helpful in understanding mesenteric traction syndrome and hopefully will be able to prevent, diagnose and appropriately treat it when I face it again. As well as, may alert my anesthesia colleagues to consider the possibility of MTS when they face unexplainable hemodynamic instability. During my literature search I used a combination of Pubmed, Mendeley, and Google scholar to find articles under the search term of mesenteric traction syndrome and subsequently used the bibliographies of these articles to deepen my search. Over the following paragraphs I will discuss the definition of mesenteric traction syndrome, the proposed etiologies of mesenteric traction syndrome, and current consensus on how to prevent and treat mesenteric traction syndrome.

\section{Discussion}

\section{Definition of mesenteric traction syndrome}

During my literature search I found multiple studies published in the late 1980s describing the phenomenon of mesenteric traction syndrome. The authors of these studies noted that traction placed on abdominal mesentery lead to four main physiologic responses: cutaneous flushing of the neck and head, a drop in mean arterial pressure with a reflexive tachycardia, and an increase in cardiac output. Prior to these initial studies, mesenteric traction syndrome had been described as a sympathetic response that resulted in splanchnic vessel vasodilation. ${ }^{1}$ These initial studies demonstrated that MTS was not a sympathetic response and was most likely mediated through a humeral prostanoid response. The most accepted definition of MTS is; hypotension, tachycardia, flushing neck and head that occurred during abdominal surgeries and in most of the instances it is shortlived phenomena.

\section{What are the mediators of mesenteric traction syndrome?}

One of the earlier studies that examined the mediators involved in MTS was published in Anesthesiology in 1988 by Seltzer et al.. The study examined 12 patients undergoing elective abdominal aortic aneurysm (AAA) repair. Preoperatively four patients were prescribed ibuprofen $12 \mathrm{mg} / \mathrm{kg}$ orally, while the other eight did not receive ibuprofen. Baseline PGF-1 metabolite of prostaglandin I2 (PGI2), levels were obtained and followed throughout the case, since prior studies had demonstrated PGI2 to be a potent vasodilator produced by the vascular endothelial cells of the mesentery and the intestinal mucosa. ${ }^{2}$ Blood levels of PGF-1were monitored pre-mesenteric traction and at 5, 10, and 30 minute intervals following traction. The study found statistically significant decreases in mean arterial blood pressure, increases in cardiac output, and increases in PGF-1 levels following mesenteric traction (MT) in patients who did not receive ibuprofen preoperatively. Patients who had received ibuprofen had no change in baseline hemodynamics or PGF-1 levels. ${ }^{3}$

One year later a study published through Cleveland clinic observed 37 patients undergoing AAA repair finding a correlation between mesenteric traction and a decrease in systolic, diastolic, and mean arterial pressure with an increase in cardiac output, cutaneous flushing, and reflexive tachycardia. The PGF-1 levels were again measured, but in addition levels of prostaglandin E2, Thromboxane B2, and histamine blood levels were measured. Following mesenteric traction there was an increase in PGF-1 levels (as shown in the previous study) without detectable changes in prostaglandin E2, Thromboxane B2, and histamine levels. Again demonstrating that PGI2 was the causative mediator of MTS. Six of the 37 patients had received aspirin prior to surgery and similar to the previous study these patients did not develop changes in hemodynamics nor in levels of mediators with MT. ${ }^{4}$

Most of the current literature published agrees that PGI2 is the main mediator of MTS, however there is one study I found that assessed the possibility of histamine release resulting in MTS. In 2002 a study conducted by Duda D et al., ${ }^{5}$ hypothesized that MTS was mediated by degranulation of mast cells resulting in histamine release. This study focused on 17 male patients undergoing AAA repair, in which half of the patients received an $\mathrm{H} 1$ receptor antagonist (dimetindene) and an $\mathrm{H} 2$ receptor antagonist (cimetidine) preoperatively while the other half of the participants received placebo. Hemodynamic parameters and histamine levels were monitored at the following intervals: 1 minute after skin incision, 5 minutes, and 20 minutes following MTS. The study found that the incidence of histamine release in the placebo group was $55 \%$ vs. $37.5 \%$ in the control group, which was not statistically significant. Between the two groups there was no significant difference in systolic blood pressure. One of the study finding was that the frequency of arrhythmias and the requirement for vasopressor medications during MT was lower in patient's receiving anti-histamines pre-operatively than in patients who were not medicated with anti-histamine. ${ }^{5}$ This finding may point out to the role of histamine in the etiology of MTS.

An editorial written by D.V. Averginos et al. in 2005 hypothesizes that the mast cell is likely the initiator of MTS, however it is due to a mix of chemical mediators such as bradykinin, serotonin, tumor necrosis factor alpha, vasoactive intestinal peptide, and vascular endothelial growth factor that results in hemodynamic instability. Currently there are no studies further assessing the role of these factors in the etiology of MTS, with current clinically proven and accepted mediator of MTS being PGI2.

\section{Diagnosis of mesenteric traction syndrome and differential diagnosis}

As described previously mesenteric traction is combination of clinical symptoms including facial flushing, a drop in mean arterial blood pressure, reflexive tachycardia, and an increase in cardiac output. On review of case reports involving the development of mesenteric traction, the diagnosis is often made by the presentation of these clinical symptoms without the aid of laboratory tests such as PGI2 levels. ${ }^{6}$ Development of a clear differential diagnosis is necessary to rule out other possible causes of acute hypotension with a reflexive tachycardia.

Adverse drug reactions and anaphylaxis are most likely to mimic the symptoms of MTS. According to a study published in the Journal of Allergy and clinical Immunology in 2016 reviewing the incidence of adverse drug reactions in anesthesia over an eight-year period, an IgE mediated drug hypersensitivity reaction most commonly presents with cardiovascular symptoms in $84 \%$ of cases which include hypotension or cardiovascular collapse, and cutaneous symptoms (70\% of cases). These symptoms can be differentiated from MTS by the presentation of angioedema (11\%), urticarial (20\%), bronchospasm $(41 \%)$, increased levels of tryptase $(45 \%)$, and previously described drug $(18 \%)$ and food intolerances $(8.5 \%)$. 
Other etiologies that would mimic the cardiovascular collapse seen with mesenteric traction syndrome include pulmonary embolism, acute coronary syndrome, inferior vena cava occlusion/ compression, and acute surgical blood loss. Both acute coronary syndrome and pulmonary embolism can be differentiated from MTS through laboratory testing and trans-esophageal echocardiography. Patients with pulmonary embolisms often present with risk factors for thromboembolism and will have elevated D-dimer at the time of the event. TEE has been shown to be accurate in up to $80 \%$ of patients with acute PE showing RV dilation, leftward movement of the intraatrial septum, and tricuspid regurgitation. ${ }^{8}$

\section{Prevention of mesenteric traction syndrome}

In 1990 a study was performed showing that pretreatment with ibuprofen prevented the release of prostacyclin during mesenteric traction resulting in stable hemodynamic parameters. The study included 27 patients undergoing AAA repair; the control group received ibuprofen pre-operatively, the placebo group received no premedication. Hemodynamic parameters and levels of PGF-1 and thromboxane B2 were measured prior to mesenteric traction and then $5,10,30$, and 45 minutes following traction. Patients who received ibuprofen did not develop statistically significant elevations in PGF1 , thromboxane B2, or hemodynamic changes. Patients who received placebo developed cutaneous flushing, reduced SVR, increased HR and $\mathrm{CI}$, and increased levels of PGF-1. The placebo group also developed late increases in levels of thromboxane B2. The authors of the study concluded thromboxane $\mathrm{B} 2$ to be a counter regulatory mechanism to the vasodilatory effects of PGI2. ${ }^{9}$ This study demonstrated that COX inhibitors could help in the prevention of MTS.

By 2000 multiple studies had demonstrated that ibuprofen/nonselective COX inhibitors helped in preventing mesenteric traction syndrome. The first prospective, double-blind randomized controlled trial was in 2006 which was published in the Journal of European Pharmacology assessed the use of COX-2 inhibitor, Parecoxib, on the prevention of MTS. Plasma levels of PGF-1were monitored along with changes in hemodynamics. The study showed that parecoxib neither significantly affected the plasma levels of PGF-1 nor caused any significant alteration in hemodynamic compromise that was associated with MTS. This study demonstrated that PGI2 release during MTS is not mediated through COX-2 inhibition and therefore COX-2 inhibitors are not effective in preventing MTS. ${ }^{10}$

In 2010, Y Nomura et al., ${ }^{11}$ published a study demonstrating that remifentanil infusions increased the likelihood of development of mesenteric traction syndrome. This study included 100 patients undergoing laparoscopic and open abdominal surgeries. For the procedure participants received either remifentinal infusions for analgesia or intravenous fentanyl. Plasma PGF-1 levels were measured prior to surgery and 20 minutes following skin incision. The study found that $40 \%$ of the patients receiving remifentanil developed MTS, while only $10 \%$ of patient's in the fentanyl group developed MTS. ${ }^{11}$ Previous studies have shown the mechanism of action of remifentanil's vasodilation is likely due to enhanced secretion of nitric oxide and prostacyclin from endothelial cells. ${ }^{12}$ Therefore it is likely that remifentanil increases the release of prostacyclin associated with MTS. This study also found that patients undergoing opened laparotomy were more likely to develop MTS than patients undergoing laparoscopic laparotomy.

A subsequent study in 2012 showed that the use of flurbiprofen axetil (intravenous prodrug that is quickly metabolized to active form by plasma esterases) could prevent the incidence of MTS in patients who received remifentanil. The study was published in the Journal of Anesthesia, it was a prospective randomized control trial including 30 patients undergoing abdominal surgery with remifentanil infusions. Half of the patients received flurbiprofen axetil pre-operatively, the other half received placebo. The incidence of MTS was $80 \%$ in the placebo group vs. $6.7 \%$ in the flurbiprofen axetil group. The use of flurbiprofen axetil resulted in an absolute risk reduction of $73 \%$ (with $95 \%$ confidence interval). ${ }^{13}$

In conclusion mesenteric traction syndrome is prevented by a mix of pharmaceutical drugs and surgical approach. Ibuprofen and other non-selective COX inhibitors, such as flurbiprofen axetil, have been shown to significantly reduce the risk of development of MTS. Avoiding remifentanil infusions also reduces the risk of MTS by avoiding remifentanil's induced excess secretion of prostacyclins and nitric oxide leading to vasodilation. Lastly the above studies have shown that open procedures versus laparoscopic procedures largely increase the risk of development of MTS.

\section{How to treat mesenteric traction syndrome}

In 1998 Brinkmann et al., ${ }^{14}$ investigated the innate counterregulatory mechanisms that stabilize hemodynamic effects caused by PGI2 release. The authors noted that in a previous study PGF1 levels remained elevated for up to 6 hours following mesenteric traction but typically the hemodynamic effects only lasted 30 minutes following mesenteric traction. ${ }^{14}$ The study included 42 patients undergoing major abdominal surgery who were either pretreated with ibuprofen or placebo. Following mesenteric traction measurements of patient's plasma concentrations of arginine vasopressin, thrombaxin B2, renin, and catecholamines were obtained. Patients in the placebo group had PGF-1 levels that were 15 times higher than the ibuprofen pretreatment group; these elevations were associated with acute hemodynamic compromise. Despite large elevations in PGF-1 patients in the placebo group regained hemodynamic stability within 30 minutes. The authors of the study hypothesized this was likely due to the observed increases in thrombaxin B2, epinephrine, renin, and arginine vasopressin following mesenteric traction. Epinephrine levels stayed elevated the longest following mesenteric traction. ${ }^{15}$ This study demonstrates that there is an innate defense mechanism to mesenteric traction which accounts for why many patients who do have mesenteric traction do not require prolonged pharmacological treatment for hemodynamic changes.

As previously mentioned, multiple studies have shown the incidence of mesenteric traction syndrome can be largely reduced by pretreatment with non-selective COX inhibitors. ${ }^{9,13,16}$ However not all patients are candidates for pretreatment with a non-selective COX inhibitor, for example patients with coagulation abnormalities or who are at risk of surgical bleeding. A study published in 2013 by M Takada et al., ${ }^{16}$ assessed the effectiveness of flurbiprofen axetil administration at the onset of MTS symptoms instead of pretreatment. The results of the study showed that administration of $50 \mathrm{mg}$ of flurbiprofen axetil intravenously at onset of MTS decreased the severity of symptoms. ${ }^{16}$ Thus a possible treatment option intraoperatively, after the development of MTS is to administer flurbiprofen axetil intravenously.

Despite the possibility of giving non-selective COX-inhibitors at the onset of MTS there is still a subset of patients that are unable to receive them (aspirin allergies or surgical bleeding). A case report from 2004 discusses the use of high dose vasoconstrictors in the treatment of severe mesenteric traction without the use of COX inhibition. The report discusses a patient undergoing a colectomy and end ileostomy for ulcerative colitis who developed severe hypotension (systolic blood 
pressure of $60 \mathrm{mmgHg}$ ) following mesenteric traction. The patient was given two doses of $100 \mathrm{mcg}$ of phenylephrine with no change in blood pressure. Subsequent boluses of $1500 \mathrm{mcg}$ phenylephrine over a 2 minute period returned the patient's blood pressure to $90 \mathrm{mmHg}$. Ultimately the patient required another $3500 \mathrm{mcg}$ of phenylephrine boluses during the 20 minutes following mesenteric traction to keep her blood pressure greater than $90 \mathrm{mmgHg} .{ }^{6}$ This case report demonstrates that MTS can be treated in the absence of cox-inhibitors with large doses of vasoconstrictors. Phenylephrine was used for this case since it was the easiest accessible vasoconstrictor, however the vasoconstrictor of choice has yet to be studied.

The most recent publication I encountered on my literature search assessed the use of FloTrac sensor monitoring of stroke volume variation (SVV) and systemic vascular resistance index (SVRI) to guide in the diagnosis and treatment of MTS. In this study by Takahashi et al. ${ }^{17}$ they noticed that $35.7 \%$ of patients who developed MTS had a SVV $>13 \%$. The study showed that about one third of patients who developed MTS would benefit from fluid therapy over vasoconstrictor therapy to improve cardiac output. This study also demonstrated that the use of SVRI was helpful in the diagnosis of MTS and proper management of the hemodynamic parameters.

\section{Conclusion}

MTS is a short-lived turbulence in hemodynamic parameters, which is in most cases has a benign outcome. However, MTS should be considered seriously in patients with multiple pre-existing comorbidities that even short-lived cardiovascular instability could put them at serious risk for stroke or an acute coronary event. In summary, mesenteric traction syndrome is caused by the release of PGI2 from vascular endothelial cells within the abdominal mesentery with the possibility of involvement of other mediates. PGI2 release results in systemic vasodilation resulting in a decrease in MAP, a reflexive tachycardia, and an increase in cardiac output. Around two thirds of these patients will also develop cutaneous flushing. There is a possibility that other mediators, possibly from mast cell degranulation are involved in this response but more studies are necessary. Histamine does not seem to play a part in mesenteric traction syndrome and the administration of antihistamines does not prevent MTS. Multiple studies have shown the preventive effects of nonselective COX inhibitors. However, the use of COX-2 inhibition does not provide protection. Remifentanil infusions increase the likelihood of developing MTS, but prophylactic flurbiprofen axetil decreases the rate of MTS with remifentanil infusion. More research is needed to look into the proper treatment of MTS when COX inhibition isn't possible (i.e. the risk of surgical bleeding and platelet dysfunction or an allergy). During my research I found a single case report that had success in treating MTS with high doses of phenylephrine. In my personal experience from the case above, high doses of epinephrine and vasopressin along with large boluses of crystalloid and colloid solutions were needed to counteract the hemodynamic instability of mesenteric traction. The use of FloTrac monitoring can help guide the anesthesiologist in the selection of vasopressor therapy versus fluid resuscitation in patients who develop severe MTS. Further research needs to be conducted to determine that vasopressor of choice in treatment of mesenteric traction, with or without the presence of COX-inhibition. Examination of the dose of non-selective COX inhibition would also be of interest in order to further decrease the risk of coagulation abnormalities.

\section{Acknowledgments}

\section{Conflicts of interest}

Authors declare that there is no conflict of interest.

\section{References}

1. Avgerinos DV, Theoharides TC. Mesenteric traction syndrome or gut in distress. Int J Immunopathol Pharmacol. 2005;18(2):195-199.

2. Balaa MA, PowellDW.Prostaglandin synthesis by enterocyte microsomes of rabbit small intestine. Prostaglandins. 1986;31(4):609-624.

3. Seltzer JL, Goldberg ME, Larijani GE, et al. Prostacylin Mediation of Vasodilation Following Mesenteric Traction. Anesthesiology. 1988;68(4):514-518.

4. Gottlieb A, Skrinska VA, O'Hara P, et al. The role of prostacyclin in the mesenteric traction syndrome during anesthesia for abdominal aortic reconstructive surgery. Ann Surg. 1989;209(3):363-367.

5. Duda D, Lorenz W, Celik I. Histamine release in mesenteric traction syndrome during abdominal aortic aneurysm surgery: prophylaxis with H1 and H2 antihistamines. Inflamm Res. 2002;51(10):495-499.

6. Woehlck H, Antapli M, Mann A. Treatment of refractory mesenteric traction syndrome without cyclooxygenase inhibitors. Anesth J Clin. 2004;16(7):542-544.

7. Mertes PM, Alla F, Tréchot P, et al. Anaphylaxis during anesthesia in France: An\&\#xa0;8-year national survey. $J$ Allergy Clin Immunol. 2016;128(2):366-373.

8. Torbicki A, Pruszynski B, Chlebus M, et al. Noninvasive Diagnosis of Suspected Severe Pulmonary Embolism: Transesophageal Echocardiography vs Spiral CT. Chest. 1997;112(3):722-728.

9. Hudson JC, Wurm WH, O'Donnel TF, et al. Ibuprofen pretreatment inhibits prostacyclin release during abdominal exploration in aortic surgery. Anesthesiology. 1990;72(3):443-449.

10. Bucher M, Kees FK, Memann B, et al. Prostaglandin I2 release following mesenteric traction during abdominal surgery is mediated by cyclooxygenase-1. Eur J Pharmacol. 2006;536(3):296-300.

11. Nomura Y, Funai Y, Fujimoto Y, et al. Remifentanil increases the incidence of mesenteric traction syndrome: Preliminary randomized controlled trial. J Anesth. 2010;24(5):669-674.

12. Ünlügenç $\mathrm{H}$, Itegin $\mathrm{M}$, ÖCal $\mathrm{I}$, et al. Remifentanil produces vasorelaxation in isolated rat thoracic aorta strips. Acta Anaesthesiol Scand. 2003;47(1):65-69.

13. Fujimoto Y, Nomura Y, Hirakawa K, et al. Flurbiprofen axetil provides a prophylactic benefit against mesenteric traction syndrome associated with remifentanil infusion during laparotomy. $J$ Anesth. 2012;26(4):490-495.

14. Brinkmann A, Seeling W, Wolf CF, et al. The Impact of Prostanoids on Pulmonary Gas Exchange During Abdominal Surgery with Mesenteric Traction. Anesth Analg. 1997;85(2):274-280.

15. Brinkmann A, Seeling W, Wolf CF, et al. Vasopressor hormone response following mesenteric traction during major abdominal surgery. Acta Anaesthesiol Scand. 1998;42(8):948-956.

16. Takada M, Taruishi C, Sudani T, et al. Intravenous flurbiprofen axetil can stabilize the hemodynamic instability due to mesenteric traction syndrome-Evaluation with continuous measurement of the systemic vascular resistance index using a flotrac ${ }^{\circledR}$ sensor. $J$ Cardiothorac Vasc Anesth. 2013;27(4):696-702.

17. Takahashi H, Shida D, Tagawa K, et al. Hemodynamics of mesenteric traction syndrome measured by FloTrac sensor. J Clin Anesth. 2016;30:46-50.

None. 